\title{
Characterizing interactions of Leptospira interrogans with proximal renal tubule epithelial cells
}

Takayoshi Yamaguchi ${ }^{1,4}$, Naomi Higa ${ }^{1}$, Nobuhiko Okura², Arina Matsumoto 1,5, Idam Hermawan', Tetsu Yamashiro ${ }^{1}$,

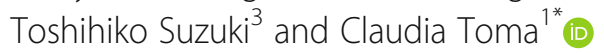

\begin{abstract}
Background: Leptospira interrogans is a pathogenic, spirochetal bacterium that is responsible for leptospirosis, an emerging worldwide zoonosis. Leptospires colonize the renal proximal tubules and chronically infect the kidney. Live bacteria are excreted into urine, contaminating the environment. While it is well known that leptospires can persist in the kidneys without signs of disease for several months, the interactions of leptospires with the proximal renal epithelial tubule cells that allow the chronic renal colonization have not been elucidated yet. In the present study, we compared the interactions between a virulent, low passage (LP) strain and a cultured-attenuated, high passage (HP) strain with renal proximal tubule epithelial cells (RPTECs) to elucidate the strategies used by Leptospira to colonize the kidney.
\end{abstract}

Results: Kinetics analysis of kidney colonization in a mouse model of chronic infection performed by quantitative real-time PCR and immunofluorescence, showed that the LP strain reached the kidney by 3 days post infection (pi) and attached to the basal membrane side of the renal epithelial cells. At 10 days pi, some leptospires were attached to the luminal side of the tubular epithelia and the number of colonizing leptospires gradually increased. On the other hand, the HP strain was cleared during hematogenous dissemination and did not colonize the kidney. Transmission electron microscopy analysis of LP-infected kidneys at 25 days pi showed aggregated leptospires and membrane vesicles attached to the epithelial brush border. Leptospiral kidney colonization altered the organization of the RPTEC brush border. An in vitro model of infection using TCMK-1 cells, showed that leptospiral infection induced a host stress response, which is delayed in LP-infected cells.

Conclusions: After hematogenous dissemination, leptospires create protective and replicative niches in the base membrane and luminal sides of the RPTECs. During the long-term colonization, leptospires attached to the RPTEC brush borders and membrane vesicles might be involved in the formation of a biofilm-like structure in vivo. Our results also suggested that the virulent strain is able to manipulate host cell stress responses to promote renal colonization.

Keywords: Leptospira interrogans, Kidney, Colonization, Proximal tubule, Renal epithelial cell

\footnotetext{
*Correspondence: claudia@med.u-ryukyu.ac.jp

'Department of Bacteriology, Graduate School of Medicine, University of the

Ryukyus, Okinawa 903-0215, Japan

Full list of author information is available at the end of the article
}

(c) The Author(s). 2018 Open Access This article is distributed under the terms of the Creative Commons Attribution 4.0 International License (http://creativecommons.org/licenses/by/4.0/), which permits unrestricted use, distribution, and reproduction in any medium, provided you give appropriate credit to the original author(s) and the source, provide a link to the Creative Commons license, and indicate if changes were made. The Creative Commons Public Domain Dedication waiver (http://creativecommons.org/publicdomain/zero/1.0/) applies to the data made available in this article, unless otherwise stated. 


\section{Background}

Leptospirosis is a widespread zoonotic disease caused by Leptospira interrogans and other pathogenic Leptospira species. The disease is epidemic in Asia, South America and Oceania but considered as an emerging infectious disease in Europe, North America and Africa [1, 2]. Leptospires enter the host through the skin or mucous membranes, disseminate hematogenously, and then reach target organs, such as the liver, lungs and, mainly, the renal proximal tubules (RPTs) in the kidneys, where they can survive for several months [3]. Leptospiremia in the patients as well as the animal hosts after infection occurs once, at the early stage of infection, for approximately 3 to 7 days [4].

In vitro studies have shown that pathogenic leptospires can evade host defense mechanisms by surviving within macrophages, delaying phagosome maturation [5], and can resist to reactive oxygen species [6]. Pathogenic leptospires can also recruit soluble complement regulators, thus preventing activation and formation of a lytic membrane attack on its surface, leading to its successful dissemination to the target organs [7]. Recently, in vivo imaging systems were used to analyze the distribution of leptospires in the whole bodies of infected animals during the early stages of infection as well as in late colonization. These studies revealed that at the early stage of infection, adipose tissue is the colonization site, where leptospires grow before hematogenous dissemination [8]. The majority of leptospires are cleared during dissemination, but a small number of bacteria that successfully evade host defense mechanisms, colonized the kidneys where they are able to replicate and persist despite being constantly shedding into the urine [9]. A wide range of mechanisms possibly involved in the ability of leptospires to survive in the kidneys, such as biofilm formation, have been suggested [10]. Live imaging of bioluminescent bacteria and quantitative real-time PCR have been used to characterize the kinetics of kidney colonization in various animal infection models $[9,11,12]$, however, the kinetics of proximal renal tubule colonization and the mechanisms that allow the long-term persistence of bacteria in the tubules remains poorly understood.

In the hamster model of infection, leptospires in the interstitial space have been reported to induce necrosis of the epithelial cells, while no epithelial cell death was observed in a mouse model of infection [13]. These differences in epithelial cells outcome might be a result of the differential host inflammatory response during chronic infection in both animal models [13]. However, pathogen-driven strategies to promote persistence in renal proximal tubule epithelial cells (RPTECs) could not be excluded.

Ratet et al. [9] have reported that the renal colonization protects leptospires from blood defenses and antibiotic treatment. In leptospirosis patients, timely treatment with antibiotics is effective and may dramatically rescue patients from multiple organ failure. On the other hand, chronic leptospirosis may also develop in humans if bacteria persist in the tubular lumen after acute leptospirosis [14]. Chronic asymptomatic leptospirosis has recently been reported in a Peruvian population and in Taiwan, suggesting that the renal colonization is not a peculiarity of some animal carriers $[14,15]$. Since antibiotic treatment would not be effective in these asymptomatic carriers, a better understanding of the interaction of leptospires with RPTECs is needed to develop novel strategies for controlling the disease. Hence, in this work we used in vivo and in vitro models of infection to elucidate the strategies used by a virulent $L$. interrogans serovar Manilae strain to interact with RPTECs and persist in the kidneys.

\section{Methods}

\section{Bacterial strains and culture}

Leptospires were cultured in Ellinghausen-McCulloughJohnson-Harris (EMJH) broth (Difco) at $30{ }^{\circ} \mathrm{C} . \quad L$. interrogans serovar Manilae strain UP-MMC-NIID isolated from the blood of a human patient with severe leptospirosis [16] was propagated in specific pathogen-free $\mathrm{C} 3 \mathrm{H} / \mathrm{HeJ}$ mice to maintain pathogenicity and used as virulent, low-passage (LP) strain. The attenuated-high passage strain (HP) strain was generated by serially culturing the LP strain in liquid EMJH more than 60 times as described previously [17]. The attenuation of $\mathrm{HP}$ was confirmed in the $\mathrm{CH} 3 / \mathrm{HeJ}$ mice infection model which is an in vivo murine model of severe leptospirosis [18].

\section{Animal infection}

L. interrogans LP or HP strains in the exponential phase of growth were adjusted to $10^{7}$ cells in $0.5 \mathrm{~mL}$ of PBS (a sublethal dose) and injected intraperitoneally to C57BL/ 6 mice (female, 5-weeks old) purchased from Japan SLC (Tokyo, Japan). Mice were acclimated for at least 1 week in the animal facility with free access to food and water. The animals ( $n=5$ per group) were sacrificed at $1,3,10$, 21 or 25 days pi by cervical dislocation and kidneys were quickly removed. One kidney was fixed in $4 \%$ formaldehyde for immunostaining and the other one was divided for use as material for DNA extraction and culture in EMJH broth to confirm the viability of colonizing leptospires. The kidney tissues were then embedded in tissue-embedding medium to ensure Optimal Cutting Temperature (O.C.T. compound, Sakura Finetek), frozen and sectioned with a Leica cryostat (model CM 1900).

\section{Quantitative real-time PCR (qPCR)}

DNeasy Blood \& Tissue Kit (Qiagen) was used for DNA extraction from the infected kidneys after mechanical 
disruption. Two microliters of DNA were added to a mixture containing Brilliant III SYBR Green QPCR master mix (Stratagene, Agilent Technologies) and $0.5 \mu \mathrm{M}$ of both forward and reverse 16S rRNA gene primers as described previously [5]. The number of total bacteria was determined using a standard curve generated by the serial dilution of genomic DNA extracted from in vitro-cultivated bacteria and was expressed as the number of leptospires per $25 \mathrm{ng}$ of total DNA extracted from kidneys.

\section{Cell culture and infection}

TCMK-1 (ATCC ${ }^{\circ}$ CCL-139 $9^{\mathrm{mi}}$ ) cells were grown in Dulbecco's Modified Eagle Medium (DMEM) supplemented with $10 \%$ fetal bovine serum (FBS). The cells were seeded at $5 \times 10^{5}$ cells per well in a six-well plate and maintained in a humidified incubator at $37{ }^{\circ} \mathrm{C}$ with $5 \% \mathrm{CO}_{2}$ for $24 \mathrm{~h}$ before infection. The cells were infected with leptospires at a multiplicity of infection (MOI) of 100 per cell in prewarmed FBS-free DMEM. In the experiments using inhibitors, the TCMK-1 cells were incubated with $20 \mu \mathrm{M}$ PARP inhibitor VIII, PJ34 (Calbiochem), $20 \mu \mathrm{M}$ Z-VAD-FMK (R\&D System) or $200 \mu \mathrm{M}$ Trolox (Sigma). The plates were centrifuged at $500 \mathrm{~g}$ for $10 \mathrm{~min}$ to synchronize the stage of infection. TCMK-1 were then incubated at $37^{\circ} \mathrm{C}$ in $5 \% \mathrm{CO}_{2}$ and fixed at the appropriate time pi for immunostaining or TUNEL staining with $2 \%$ paraformaldehyde overnight at $4{ }^{\circ} \mathrm{C}$. For crystal violet staining, cells were fixed with $4 \%$ paraformaldehyde at room temperature. For detection of reactive oxygen species, CellROX Green Reagent (Molecular Probes) was added to infected cells and incubated for $30 \mathrm{~min}$ for image acquisition using the Leica AF6500 widefield microscope.

\section{Immunofluorescence microscopy}

Rhodamin-phalloidin (Molecular Probes) was used to visualized the actin filaments and TO-PRO-3 (Molecular Probes) was used to visualized the DNA. All secondary antibodies used in this study were from Jackson ImmunoResearch. Leptospires were stained with a rabbit polyclonal anti-L. interrogans antiserum (kindly provided by SYAM Villanueva, University of the Philippines) and labeled with anti-rabbit FITC, TRITC or Alexa647. For cubilin immunostaining, acetone-fixed sections were stained after epitope retrieval by heating in citrate buffer (10 mM sodium citrate [pH 6.0]), with anti-cubilin (R\&D Systems, AF3700, sheep polyclonal) followed by the tyramide amplification method (TSA-Plus Fluorescein System; Perkin Elmer Life Sciences). Anti-AIF (Cell Signaling, D39D2 XP Rabbit mAb) was labeled with FITC-labeled secondary antibody. Stained samples were observed using a confocal laser scanning microscope (Leica TCS-SPE).

\section{Transmission electron microscopy (TEM)}

For TEM analysis kidneys were pre-fixed in $2.5 \%$ glutaraldehyde in $0.1 \mathrm{M}$ sodium cacodylate buffer ( $\mathrm{pH} 7.4$ ), and then were post-fixed in $1 \% \mathrm{OsO} 4$ in the same buffer. The tissues were dehydrated in a graded ethanol series and embedded in epoxy resin (TAAB812). Thin sections were cut on an ultra-microtome with a diamond knife (MT-2C, RMC). The sections were double stained with uranyl acetate and lead citrate and examined with an electron microscope at $80 \mathrm{kV}$ (H-750, Hitachi), at the University of the Ryukyus, Research Laboratory Center.

\section{Cell death assays}

For the crystal violet assay, fixed cells were stained with $0.1 \%$ crystal violet solution. After extensive washing, the dye was eluted and analyzed at $595 \mathrm{~nm}$. The TUNEL assay was performed with the Dead-End fluorometric TUNEL system (Promega) as recommended by the manufacturer.

\section{Statistical analyses}

Statistical analyses were performed by the unpaired two-tailed Student's $t$ tests. Differences were considered significant at $P<0.05$.

\section{Results}

Kinetics of leptospiral dissemination in mouse infected with low-passage number (LP) virulent or high-passagenumber (HP) attenuated Leptospira interrogans strains

The kinetics of leptospiral dissemination was studied in C57BL/6 mice after intraperitoneal (IP) injection of a sublethal dose of LP or HP L. interrogans strains [17]. Consistent with previous reports $[9,11]$, the virulent LP strain disseminated and reached the kidney at 3 days post- infection (pi). The bacterial DNA load in the kidney gradually increased during the course of infection as observed by qPCR (Fig. 1a) and tubules were colonized by 25 days pi as observed by immunofluorescence (Fig. 1b). Although a hamster model of infection showed that IP injection of an HP L. interrogans strain resulted in the dissemination of leptospires to the kidney and colonization by 7 days pi [11], our results suggested that the HP strain was almost completely cleared during hematogenous dissemination in the mouse model of chronic infection (Fig. 1a). Histological observations of renal tissues from LP-infected mouse at 25 days pi showed normal glomerulus and tubules (Fig. 1c), which is consistent with a previous reported that compared chronic leptospirosis in hamsters and mouse models and showed no evolution of lesions in mouse kidneys [13].

Interstitial leptospires translocated to the tubular lumen The kinetics of bioluminescent leptospires determined by live imaging analysis in mice suggest that the 

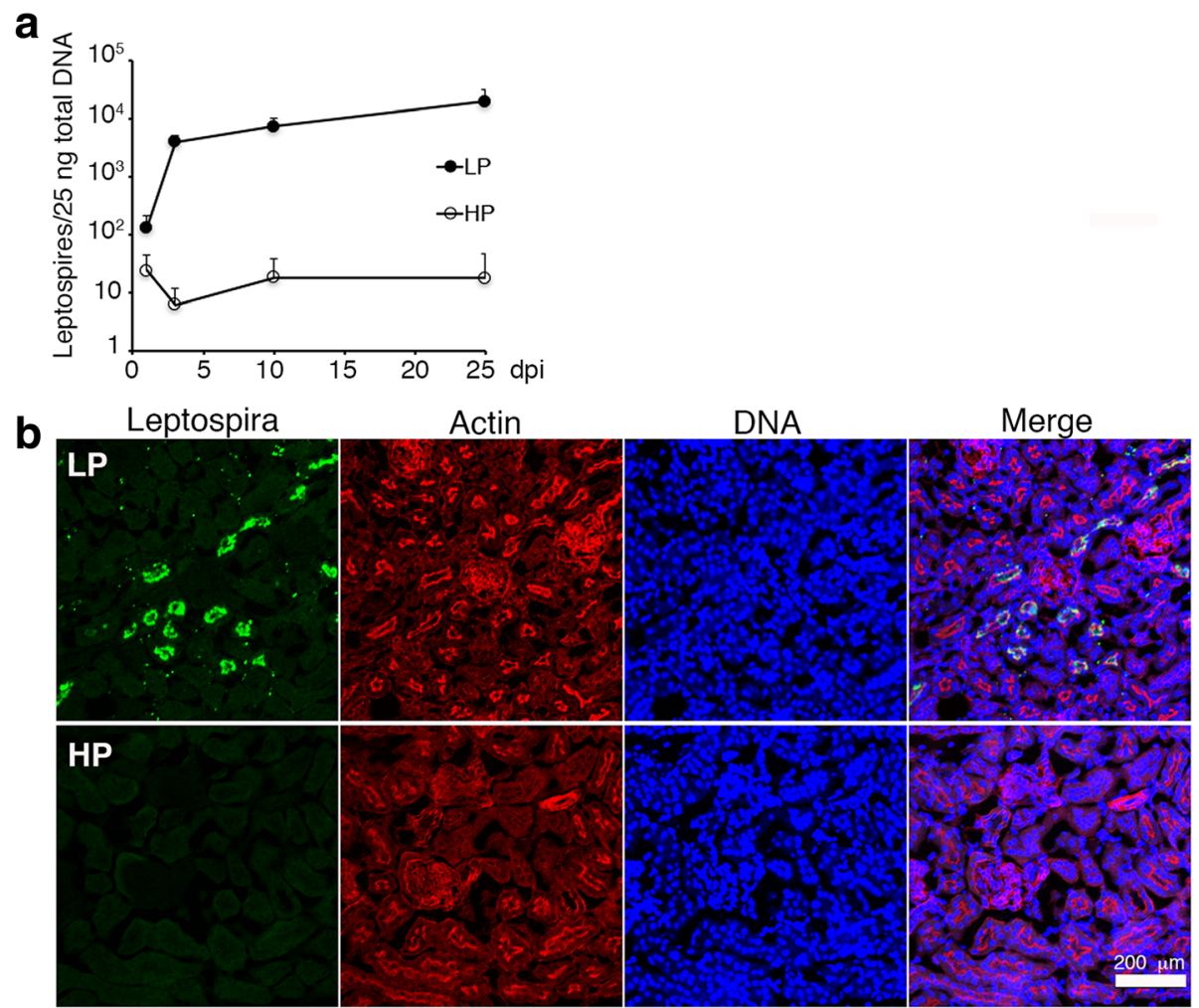

C

non infected

LP

$\mathrm{HP}$

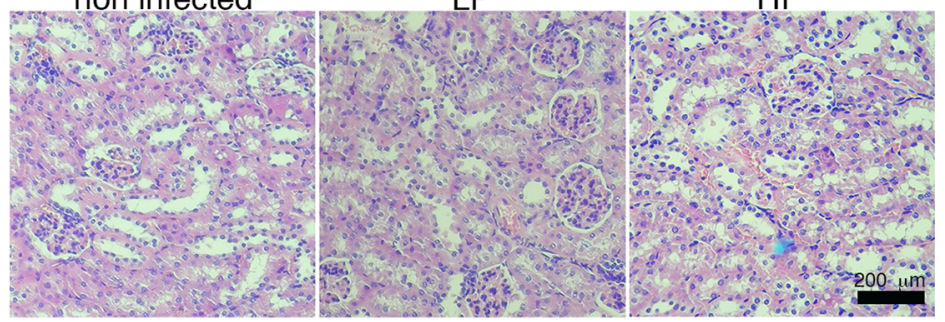

Fig. 1 Virulent low passage (LP) strain colonizes the kidney, while high passage strain (HP) is cleared during hematogenous dissemination. a LP or HP strain were used to infect 5 weeks-old C57BL/6 mice. A sublethal dose of exponential growing bacteria was inoculated intraperitoneally and the presence of leptospiral DNA in the kidneys was monitored by qPCR at 1, 3, 10 and 25 days pi. $\mathbf{b}$ Immunofluorescence staining of LP- and HP-infected kidney at 25 days pi. Bacteria were detected with FITC-labeled secondary antibodies (green), actin were labeled with rhodaminephalloidin (red) and DNA with TO-PRO-3 (blue). The merged images are also shown. $\mathbf{c}$ Histological features of kidneys from LP or HP-infected mice were compared with non-infected kidney at 25 days pi by hematoxylin and eosin staining

establishment of Leptospira sp. in the kidney is an early event, occurring in the very first days pi [9], although the kinetics at the renal tubule level are not well understood. We used immunofluorescence staining to identify leptospires in the kidneys of infected animals and quantified colonized tubules at 3,10 and 21 days pi. As shown in Fig. 2a, leptospires reached the kidney at 3 days pi; bacteria were observed as single spirochetes, attached in small numbers at the basal cytoplasm of the renal tubules or aggregated in the interstitium. At 10 days pi, some leptospires were attached to the apical side of the RPTECs and by 21 days pi, there was an increase in the number of colonized tubules as well as an increase in the bacterial load in each colonized tubule (Fig. 2a and b). These results collectively suggested that during chronic infection, interstitial leptospires translocate to the tubular lumen.

\section{Attachment of leptospires to the epithelial brush border}

To characterize the Leptospira-colonized tubules we dually immunostained the infected kidneys with cubilin (a receptor expressed in RPTECs) and bacteria and performed transmission electron microscopic (TEM) analysis of the infected kidneys. As shown in Fig. 3, leptospires gradually colocalized with cubilin during the course of the infection. TEM analysis of kidney sections showed that the tubular lumen was completely filled with aggregated leptospires interacting with each other directly or through membrane vesicles. Heavily colonized epithelial cells did 


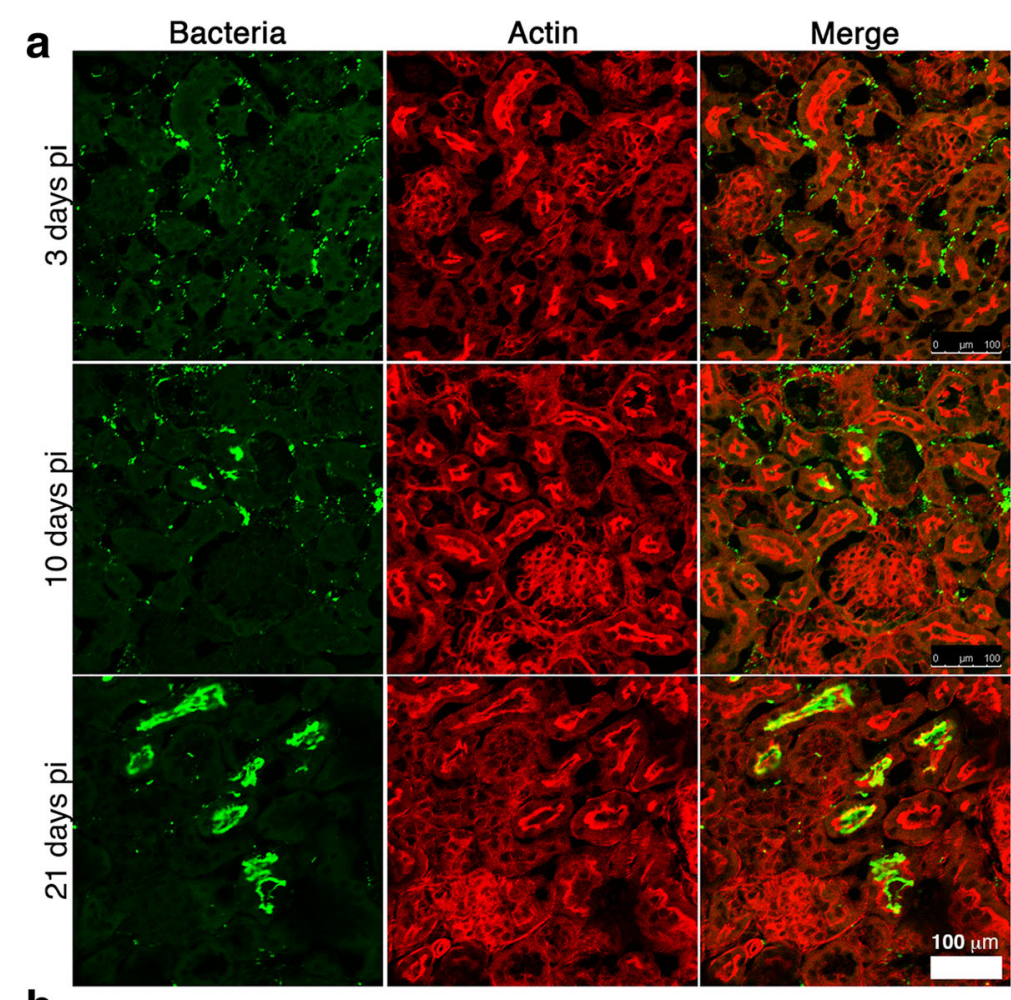

b

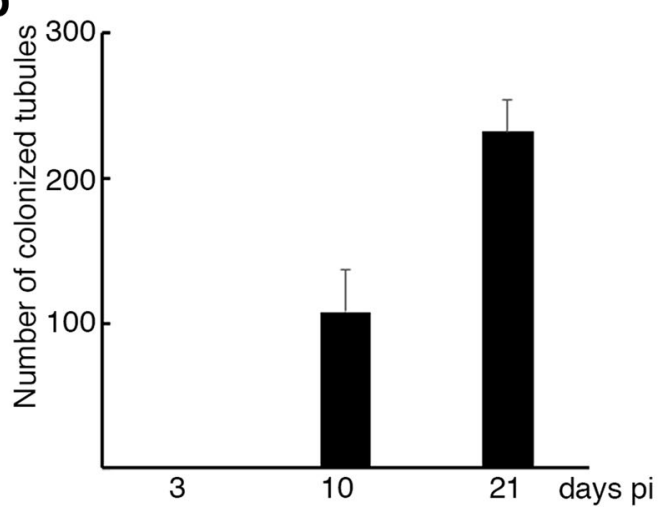

Fig. 2 Kinetics of leptospiral dissemination in mouse infected with LP strain. Five weeks-old C57BL/6 mice were i.p. infected with a sublethal dose of exponentially growing LP strain and the kidneys were fixed at 3,10 and 21 days pi and processed for immunofluorescence. Leptospires were stained with FITC-labeled secondary antibodies (green) and actin were labeled with rhodamine-phalloidin (red). a Representative confocal images. b The number of colonized renal tubules were quantified in 10 fields of view at $\times 10$ magnification. Data are presented as the mean \pm SD of three independent experiments

not show any sign of cell death, but the organization of the brush border in LP-infected kidneys was altered when compared with HP-infected kidney (Fig. 4a and b). Collectively, these results suggested that during tubular colonization, aggregated leptospires formed a biofilm-like structure to maintain a protective and replicative niche in a limited number of RPTs.

\section{Delayed oxidative stress response in LP-infected cells} The adherence of exogenous components, such as crystals, to RPTECs during kidney stone disease, can cause various cellular reponses including the production of reactive oxygen species (ROS) and the stimulation of signaling molecules [19]. To better understand the effect of Leptospira infection on RPTECs, we used an in vitro model of RPTEC infection. Infection of the mouse RPTEC line, TCMK1, showed that adherence of leptospires to RPTECs induced an increase in intracellular ROS (Fig. 5a) and cleavage of DNA as shown by TUNEL staining (Fig. $5 \mathrm{~b}$ and $\mathrm{c}$ ). This oxidative stress response was delayed in LP-infected cells. 


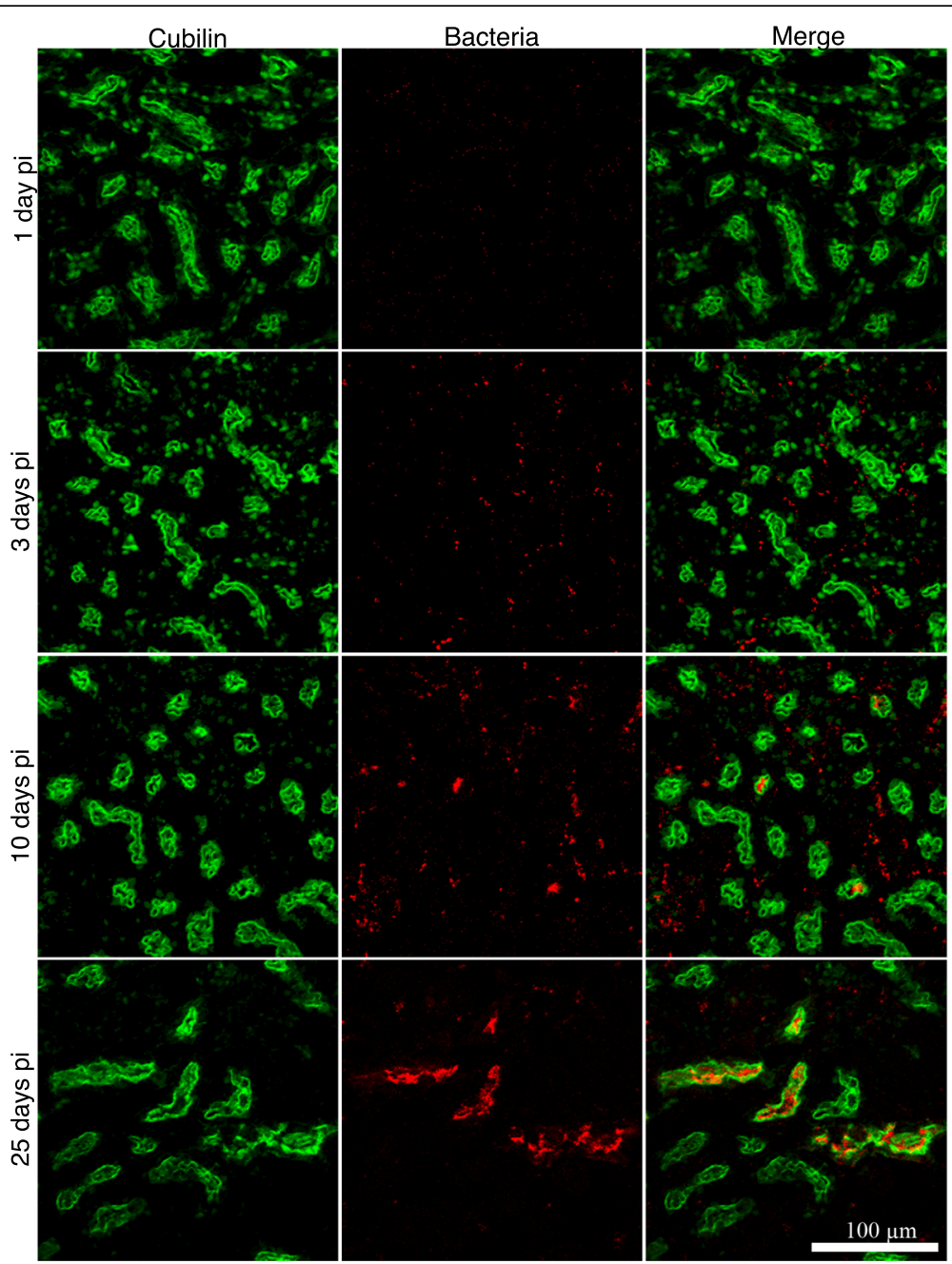

Fig. 3 Virulent Leptospira colonizes a limited number of proximal renal tubules. C57BL/6 mice were infected with LP strain and the kidneys were fixed at 1, 3, 10 and 25 days pi. Cubilin (green) was stained with the TSA-Plus Fluorescein System and leptospires were stained with Alexa Fluor 647-labeled secondary antibodies (red). Representative confocal images are shown

Oxidative stress can cause DNA breaks which induce the activation of the DNA nick sensor enzyme poly(ADP-ribose) polymerase-1 (PARP-1). PARP-1 is an activator of caspase-independent cell death. Overactivation of PARP-1 initiates a nuclear signal that propagates to mitochondria and induces the release of the apoptosis-inducing factor (AIF) [20]. Thus, we next investigated if PJ34 [a Poly (ADP-ribose) polymerase-1, PARP-1 inhibitor] or ZVAD-FMK (a pan-caspase inhibitor) can inhibit cell death induced by HP. As shown in Fig. 6a, cell death was inhibited by PJ34 and partially inhibited by ZVAD-FMK. Cell death was also inhibited by the anti-oxidative compound TROLOX (Fig. 6a). Moreover, the nuclear translocation of mitochondrial AIF was observed in HP-infected cells that was inhibited by PJ34 (Fig. 6b). These results suggested that leptospiral infection of RPTECs induced a host stress response which involves PARP-1 activation, followed by AIF nuclear translocation and cell death. However, virulent leptospires possess some mechanism/s to counteract this host response to create a safe niche for their long-term colonization.

\section{Discussion}

This is the first study to analyze the kinetics of leptospiral colonization of the proximal renal tubule in a mouse model of chronic infection. C57BL/6 animal model was used in this study as a chronic model of infection because it was shown by Ratet et al. to exhibit biphasic disease with a self-resolving hematogenous dissemination followed by renal colonization [9]. Thus, this model is suitable to study renal colonization mechanisms. Moreover, the availability of knocked out mice on a C57BL6/J background will allow the analysis of host factors involved 


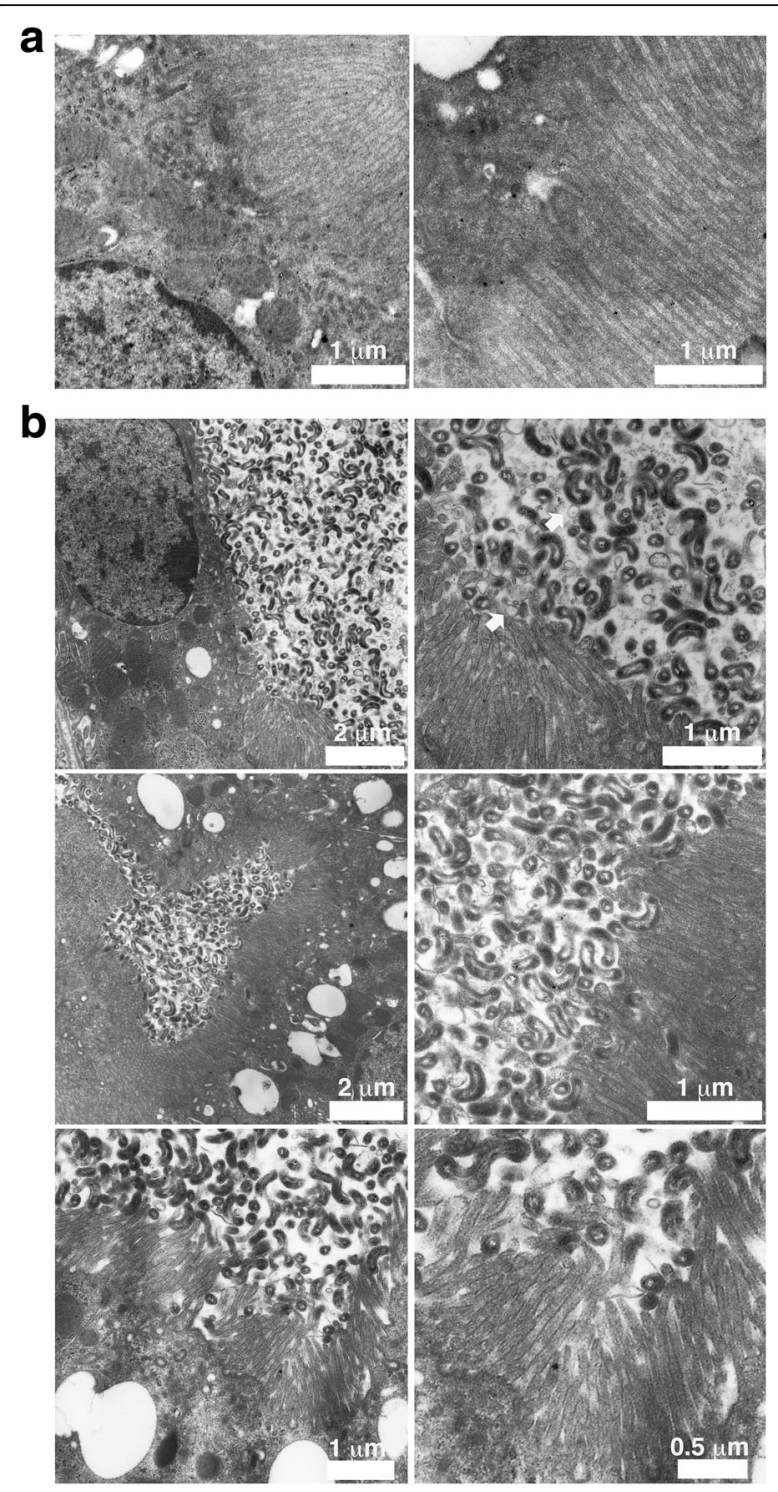

Fig. 4 Attachment of leptospires to the epithelial brush border. C57BL/6 mice were infected with either HP (a) or LP strain (b), the kidneys were fixed at 25 days pi and processed for transmission electron microscopy. Arrows indicate membrane vesicles

in kidney colonization in the future. Our results suggested that leptospires become attached at the basal cytoplasm of RPTECs before migrating from the interstitial space to the lumen side. These results are in agreement with previous observations by Marshall [21] and Barocchi et al., that suggested that leptospires rapidly translocated in polarized epithelial cell monolayers [22]. By 25 days pi leptospires reside mainly in cubilin-positive RPTs. Interestingly, the number of colonized tubules is limited and some tubules are devoid of leptospires. Our results suggested that the activation of signals that allow the bacterial replication and tubular colonization requires a threshold of leptospiral infective dose in each RPT.

Biofilms are structured communities of adherent microorganisms composed of a complex extrapolymeric substance matrix, DNA and membrane vesicles [23]. Biofilms represent a microbial survival strategy, where microorganisms exist in a dynamic equilibrium to form clusters of bacteria [24]. Saprophytic and pathogenic leptospires are able to form biofilms in vitro and it was suggested that this ability might help the bacteria to survive in environmental habitats and to colonize the hosts [25]. In vivo leptospiral aggregates were observed in placental vessels from infected pregnant guinea pigs [26]. On the other hand, membrane vesicles production by Leptospira have been reported in vitro in response to environmental stressor [27-29]. Our TEM analysis showed the presence of MVs in a biofilm-like structure within the tubules. Environmental changes in the renal tubules such as the release of host factors and low iron, induce the transition to a biofilm lifestyle that can cause the modulation of MV production [30]. Therefore, our observation suggests that to survive within tubules and/ or interact with RPTECs leptospires are induced to produce a biofilm-like structure. Recently, Turnbull et al. reported that explosive cell lysis is a mechanism for the biogenesis of bacterial membrane vesicles and biofilms in Pseudomonas aeruginosa [23]. Thus, the MVs that we observed might also be remnants of dead leptospires. Although detachment of bacteria from biofilms has been considered as a passive behavior for long time, some reports suggested that it can be an active process that allow bacteria to colonize new niches before nutrients become limited [24]. Intermittent excretion of leptospires into the urine in chronically infected animals might be the result of biofilm detachment from the tubules and the excretion of bacteria as a complex aggregate might confer advantages to survive in the environment. Further studies will elucidate the possible association between biofilm formation in renal tubules and the pathogenic cycle of Leptospira.

The comparison of LP and HP Leptospira strains has been used to identify virulence-associated genes in several studies [17, 31-33]. Ballard et al. reported that virulent leptospires attached to RPTECs, while the avirulent variant of a serovar Copenhageni strain did not adhere to epithelial cells at all [34]. In this study, we found that the avirulent variant of a serovar Manilae strain adhere to RPTECs. Therefore, our data suggested that the capacity to adhere to epithelial cells is not correlated with virulence.

Although HP was cleared during hematogenous dissemination in the chronic model of infection used in this study, the infection of TCMK1 cells suggested that the manipulation of host oxidative stress response is a 

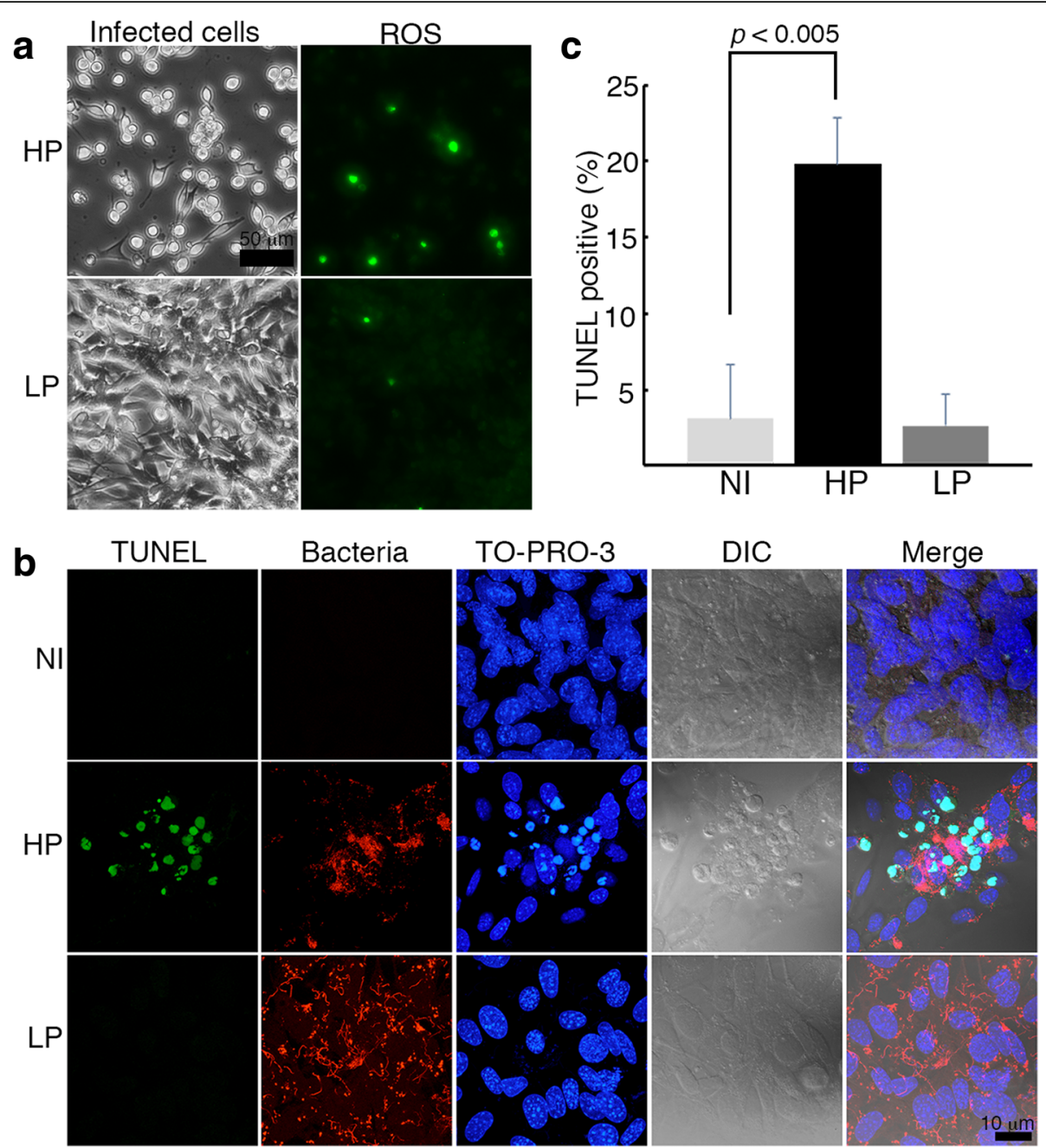

Fig. 5 Delayed of ROS production in LP-infected cells. TCMK-1 cells were infected either with LP or HP strain. a Detection of intracellular ROS. The nuclear green fluorescence reflects ROS. (b and c) Non-infected (NI) and infected cells were fixed and processed for Leptospira immunostaining and TUNEL staining (green) at $48 \mathrm{~h}$ pi. b Representative confocal images. Total bacteria were detected with TRITC-labeled secondary antibodies. DNA was stained with TO-PRO-3 (blue). The merged image of immunofluorescence and differential interference contrast (DIC) are also shown. $\mathbf{c}$ Quantification of TUNEL positive cells. Data are the mean \pm SD of three independent experiments

mechanism used by the virulent strain to maintain a protective and replicative niche in RPTs during chronic infection of kidneys. Microbial infection can trigger several host cell responses such as oxidative stress, mitochondrial stress and DNA stress which activate antimicrobial defense systems. The activation of these stress responses can also lead to cell death [35]. However, pathogenic bacteria are able to manipulate the host cell reponses in order to promote the long-term colonization of epithelial cells [36, 37]. Oxidative stress that occurs during the host defense response following infection with Leptospira spp. have been reported to be regulated by the peroxide sensor and the transcriptional regulator PerR [38, 39]. Moreover, catalase is required for the virulence of Leptospira spp. in an acute model of infection [6], suggesting that the inhibition of oxidative stress response might be a strategy used by Leptospira in the acute as well as the late stage of infection. We found that cell induction by HP leading to nuclear translocation of AIF is PARP-1 dependent. The release of mitochondrial AIF has been reported as a mechanism of Leptospira-induced apoptosis in macrophages through a caspase-8- dependent pathway [40]. Thus, different signal pathways are activated in response to Leptospira-infection during the course of infection and the understanding of the interactions between Leptospira and the different cell types will help to achieve a better control of leptospirosis. 


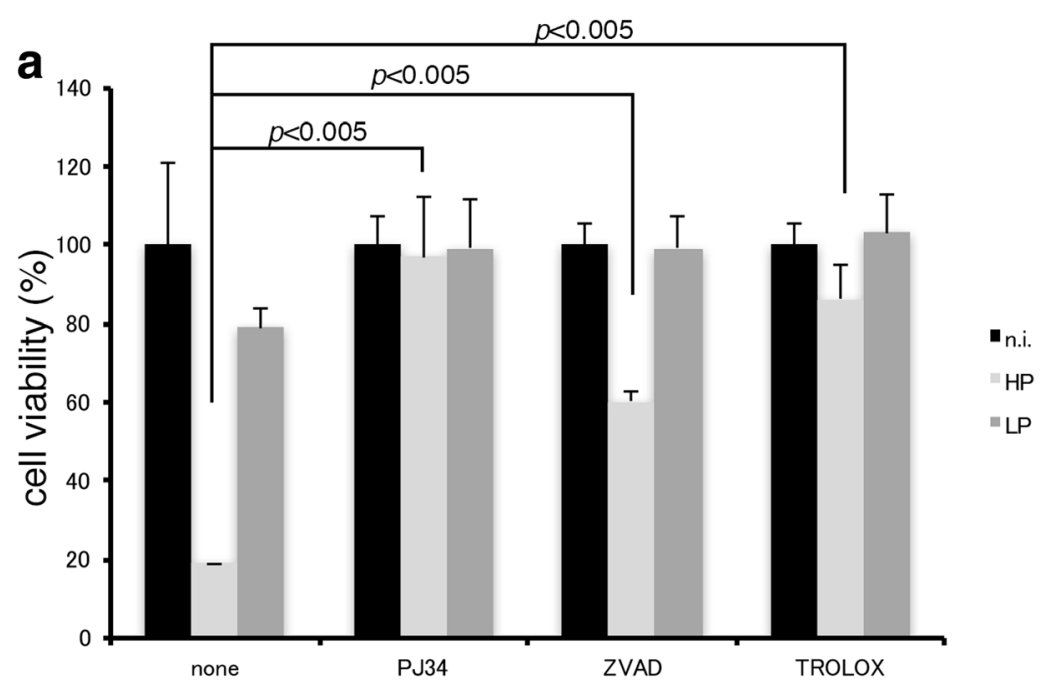

b

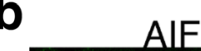

AlF

DNA

DIC

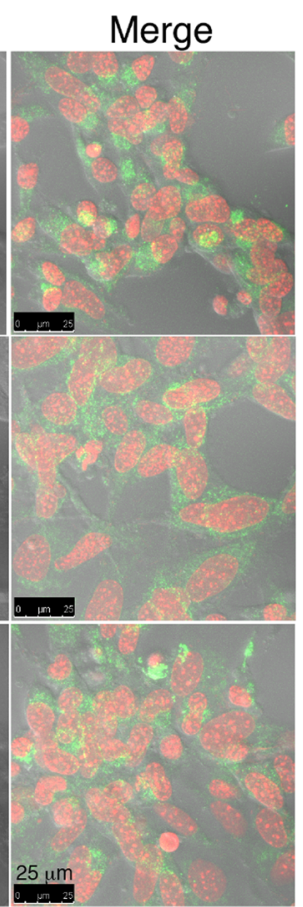

Fig. 6 Inhibition of the oxidative stress response confers protection to HP-infected TCMK-1 cells. TCMK-1 cells were pre-treated with different inhibitors and infected with either HP or LP strain for 48 h. a Cell viability was determined by crystal violet assay and shown as the mean percentages \pm SD of three independent experiments. $\mathbf{b}$ Cells were fixed and stained for AlF. Representative confocal images shown nuclear translocation of mitochondrial AIF in HP-infected cells, which is inhibited by the PARP-1 inhibitor, PJ34

\section{Conclusion}

Our results suggested that after escaping the blood defenses, leptospires create protective and replicative niches in the base membrane and luminal sides of RPTECs by manipulating the host oxidative stress response and forming a biofilm-like structure. During the long-term colonization, leptospires aggregated and attached to the brush border and membrane vesicles which are involved in the formation of a biofilm-like structure.

\section{Abbreviations}

AIF: Apoptosis-inducing factor; HP: High passage; LP: Low passage; PARP1: Poly (ADP-ribose) polymerase-1; pi: Post infection; GPCR: Quantitative realtime PCR; RPTEC: Renal proximal tubule epithelial cell; TEM: Transmission electron microscopy

\section{Acknowledgements}

The authors would like to acknowledge the members of the "Research on development of control strategy for vector borne diseases and zoonosis in Okinawa and formulation for research networking" Project for their support and advice. We also thank Giichi Takaesu for advice during cell death analysis. 


\section{Funding}

This work was supported by a grant of the Okinawa Prefectural Government (CT), JSPS KAKENHI Grant Number JP15K08467 (CT), the Uruma Fund for the Promotion of Science (CT) and a grant for Strategic Research Project of the University of the Ryukyus (TY2). Each funding body has not role in the design of the study and collection, analysis, and interpretation of data and in writing the manuscript.

\section{Availability of data and materials}

All data generated or analyzed during this study are included in this published article. Datasets used in the current study are available upon request.

\section{Authors' contributions}

$\mathrm{CT}$ and TS conceived and designed the experiments; TY1, NH, AM, IH and CT performed the experiments. NO performed the transmission electron microscope analysis. TS and TY2 contributed reagents/materials/analysis tools. CT wrote the first draft of the manuscript. All authors contributed to data analysis and interpretation of results. All authors read and approved the final manuscript.

\section{Ethics approval and consent to participate}

All animal experiments were conducted in accordance with the guidelines of the University of the Ryukyus for the care and use of laboratory animals and experimental protocols were approved by the ethics committee for animal experiments of the University of the Ryukyus, Okinawa, Japan (Permit Numbers: 6043 and A2016009).

\section{Consent for publication}

Not applicable.

\section{Competing interests}

The authors declare that they have no competing interests.

\section{Publisher's Note}

Springer Nature remains neutral with regard to jurisdictional claims in published maps and institutional affiliations.

\section{Author details}

${ }^{1}$ Department of Bacteriology, Graduate School of Medicine, University of the Ryukyus, Okinawa 903-0215, Japan. ²Department of Molecular Anatomy, Graduate School of Medicine, University of the Ryukyus, Okinawa 903-0215, Japan. ${ }^{3}$ Department of Bacterial Pathogenesis, Infection and Host Response, Graduate School of Medicine and Dental Sciences, Tokyo Medical and Dental University, Tokyo 113-8510, Japan. ${ }^{4}$ Present address: Department of Food and Nutrition Science, Junior College, Sagami Women's University, Sagamihara, Kanagawa 252-0383, Japan. ${ }^{5}$ Present address: Okinawa Industrial Technology Center, Okinawa 904-2234, Japan.

\section{Received: 28 February 2018 Accepted: 20 June 2018}

\section{Published online: 04 July 2018}

\section{References}

1. Costa F, Hagan JE, Calcagno J, Kane M, Torgerson P, Martinez-Silveira MS, Stein C, Abela-Ridder B, Ko Al. Global morbidity and mortality of leptospirosis: a systematic review. PLoS Negl Trop Dis. 2015;9(9):e0003898.

2. Dupouey J, Faucher B, Edouard S, Richet H, Kodjo A, Drancourt M, Davoust B. Human leptospirosis: an emerging risk in Europe? Comp Immunol Microbiol Infect Dis. 2014:37(2):77-83.

3. Picardeau M. Virulence of the zoonotic agent of leptospirosis: still terra incognita? Nat Rev Microbiol. 2017;15(5):297-307.

4. Haake DA, Levett PN. Leptospirosis in humans. In: Adler B, editor. Leptospira and leptospirosis Curr Top in Microbiol Immunol. New York: Springer; 2015. p. 65-98.

5. Toma C, Okura N, Takayama C, Suzuki T. Characteristic features of intracellular pathogenic Leptospira in infected murine macrophages. Cell Microbiol. 2011;13(11):1783-92.

6. Eshghi A, Lourdault K, Murray GL, Bartpho T, Sermswan RW, Picardeau M, Adler B, Snarr B, Zuerner RL, Cameron CE. Leptospira interrogans catalase is required for resistance to $\mathrm{H}_{2} \mathrm{O}_{2}$ and for virulence. Infect Immun. 2012;80(11):3892-9.
7. Siqueira GH, Atzingen MV, de Souza GO, Vasconcellos SA, Nascimento AL. Leptospira interrogans Lsa23 protein recruits plasminogen, factor $\mathrm{H}$ and C4BP from normal human serum and mediates C $3 \mathrm{~b}$ and $\mathrm{C} 4 \mathrm{~b}$ degradation. Microbiology. 2016;162(2):295-308

8. Ozuru R, Saito M, Kanemaru T, Miyahara S, Villanueva SY, Murray GL, Adler B, Fujii J, Yoshida Sl. Adipose tissue is the first colonization site of Leptospira interrogans in subcutaneously infected hamsters. PLoS One. 2017;12(2): e0172973.

9. Ratet G, Veyrier FJ, Fanton d'Andon M, Kammerscheit X, Nicola MA, Picardeau M, Boneca IG, Werts C. Live imaging of bioluminescent Leptospira interrogans in mice reveals renal colonization as a stealth escape from the blood defenses and antibiotics. PLoS Negl Trop Dis. 2014;8(12):e3359.

10. Monahan AM, Callanan JJ, Nally JE. Review paper: host-pathogen interactions in the kidney during chronic leptospirosis. Vet Pathol. 2009; 46(5):792-9.

11. Wunder EA Jr, Figueira CP, Santos GR, Lourdault K, Matthias MA, Vinetz JM, Ramos E, Haake DA, Picardeau M, Dos Reis MG, et al. Real-time PCR reveals rapid dissemination of Leptospira interrogans after intraperitoneal and conjunctival inoculation of hamsters. Infec and Immun. 2016:84(7):2105-15.

12. Gomes-Solecki M, Santecchia I, Werts C. Animal models of leptospirosis: of mice and hamsters. Front Immunol. 2017;8:58.

13. Matsui M, Roche L, Geroult S, Soupe-Gilbert ME, Monchy D, Huerre M, Goarant C. Cytokine and chemokine expression in kidneys during chronic leptospirosis in reservoir and susceptible animal models. PLoS One. 2016; 11(5):e0156084

14. Yang HY, Hung CC, Liu SH, Guo YG, Chen YC, Ko YC, Huang CT, Chou LF, Tian YC, Chang MY, et al. Overlooked risk for chronic kidney disease after leptospiral infection: a population-based survey and epidemiological cohort evidence. PLoS Negl Trop Dis. 2015;9(10):e0004105

15. Ganoza CA, Matthias MA, Saito M, Cespedes M, Gotuzzo E, Vinetz JM. Asymptomatic renal colonization of humans in the peruvian Amazon by Leptospira. PLoS Negl Trop Dis. 2010;4(2):e612.

16. Koizumi N, Watanabe $\mathrm{H}$. Leptospiral immunoglobulin-like proteins elicit protective immunity. Vaccine. 2004;22(11-12):1545-52.

17. Toma C, Murray GL, Nohara T, Mizuyama M, Koizumi N, Adler B, Suzuki T. Leptospiral outer membrane protein LMB216 is involved in enhancement of phagocytic uptake by macrophages. Cell Microbiol. 2014;16(9):1366-77.

18. Viriyakosol S, Matthias MA, Swancutt MA, Kirkland TN, Vinetz JM. Toll-like receptor 4 protects against lethal Leptospira interrogans serovar icterohaemorrhagiae infection and contributes to in vivo control of leptospiral burden. Infec and Immun. 2006;74(2):887-95.

19. Fong-Ngern K, Vinaiphat A, Thongboonkerd V. Microvillar injury in renal tubular epithelial cells induced by calcium oxalate crystal and the protective role of epigallocatechin-3-gallate. FASEB J. 2017;31(1):120-31.

20. Hong SJ, Dawson TM, Dawson VL. Nuclear and mitochondrial conversations in cell death: PARP-1 and AIF signaling. Trends Pharmacol Sci. 2004;25(5):259-64.

21. Marshall RB. The route of entry of leptospires into the kidney tubule. J Med Microbiol. 1976;9(2):149-52.

22. Barocchi MA, Ko Al, Reis MG, McDonald KL, Riley LW. Rapid translocation of polarized MDCK cell monolayers by Leptospira interrogans, an invasive but nonintracellular pathogen. Infect and Immun. 2002;70(12):6926-32.

23. Turnbull L, Toyofuku M, Hynen AL, Kurosawa M, Pessi G, Petty NK, Osvath SR, Carcamo-Oyarce G, Gloag ES, Shimoni R, et al. Explosive cell lysis as a mechanism for the biogenesis of bacterial membrane vesicles and biofilms. Nat Commun. 2016;7:11220.

24. Hall-Stoodley L, Stoodley P. Biofilm formation and dispersal and the transmission of human pathogens. Trends Microbiol. 2005;13(1):7-10.

25. Ristow $P$, Bourhy $P$, da Cruz McBride FW, Figueira CP, Huerre M, Ave P, Girons IS, Ko Al, Picardeau M. The OmpA-like protein Loa22 is essential for leptospiral virulence. PLoS Pathog. 2007;3(7):e97.

26. Brihuega B, Smartino L, Auteri C, Venzano A, Caimi K. In vivo cell aggregations of a recent swine biofilm-forming isolate of Leptospira interrogans strain from Argentina. Rev Arg Microbiol. 2012;44:138-43.

27. Henry R, Lo M, Khoo C, Zhang H, Boysen Rl, Picardeau M, Murray GL, Bulach DM, Adler B. Precipitation of iron on the surface of Leptospira interrogans is associated with mutation of the stress response metalloprotease HtpX. Appl Environ Microbiol. 2013;79(15):4653-60.

28. Nally JE, Whitelegge JP, Aguilera R, Pereira MM, Blanco DR, Lovett MA. Purification and proteomic analysis of outer membrane vesicles from a clinical isolate of Leptospira interrogans serovar Copenhageni. Proteomics. 2005;5(1):144-52. 
29. Iraola G, Spangenberg L, Lopes Bastos B, Grana M, Vasconcelos L, Almeida A, Greif G, Robello C, Ristow P, Naya H. Transcriptome sequencing reveals wide expression reprogramming of basal and unknown genes in Leptospira biflexa biofilms. mSphere. 2016;1 (2):e00042-16.

30. Orench-Rivera N, Kuehn MJ. Environmentally controlled bacterial vesiclemediated export. Cell Microbiol. 2016;18(11):1525-36.

31. Satou K, Shimoji M, Tamotsu H, Juan A, Ashimine N, Shinzato M, Toma C, Nohara T, Shiroma A, Nakano K, et al. Complete genome sequences of lowpassage virulent and high-passage avirulent variants of pathogenic Leptospira interrogans serovar Manilae strain UP-MMC-NIID, originally isolated from a patient with severe leptospirosis, determined using PacBio single-molecule real-time technology. Genome Announc. 2015;3(4):e00882-15.

32. Lehmann JS, Corey VC, Ricaldi JN, Vinetz JM, Winzeler EA, Matthias MA. Whole genome shotgun sequencing shows selection on Leptospira regulatory proteins during in vitro culture attenuation. Am J Trop Med Hyg. 2016;94(2):302-13.

33. Lehmann JS, Fouts DE, Haft DH, Cannella AP, Ricaldi JN, Brinkac L, Harkins D, Durkin S, Sanka R, Sutton G, et al. Pathogenomic inference of virulence-associated genes in Leptospira interrogans. PLoS Negl Trop Dis. 2013;7(10):e2468.

34. Ballard SA, Williamson M, Adler B, Vinh T, Faine $S$. Interactions of virulent and avirulent leptospires with primary cultures of renal epithelial cells. J Med Microbiol. 1986;21:59-67.

35. Ashida H, Mimuro H, Ogawa M, Kobayashi T, Sanada T, Kim M, Sasakawa C. Cell death and infection: a double-edged sword for host and pathogen survival. J Cell Biol. 2011;195(6):931-42.

36. Ashida H, Kim M, Sasakawa C. Manipulation of the host cell death pathway by Shigella. Cell Microbiol. 2014;16(12):1757-66.

37. Mimuro H, Suzuki T, Nagai S, Rieder G, Suzuki M, Nagai T, Fujita Y, Nagamatsu K, Ishijima N, Koyasu S, et al. Helicobacter pylori dampens gut epithelial self-renewal by inhibiting apoptosis, a bacterial strategy to enhance colonization of the stomach. Cell Host Microbe. 2007;2(4):250-63.

38. Lo M, Murray GL, Khoo CA, Haake DA, Zuerner RL, Adler B. Transcriptional response of Leptospira interrogans to iron limitation and characterization of a PerR homolog. Infect Immun. 2010;78(11):4850-9.

39. Caimano MJ, Sivasankaran SK, Allard A, Hurley D, Hokamp K, Grassmann AA, Hinton JC, Nally JE. A model system for studying the transcriptomic and physiological changes associated with mammalian host-adaptation by Leptospira interrogans serovar Copenhageni. PLoS Pathog. 2014;10(3):e1004004.

40. Hu W-L, Dong H-Y, Li Y, Ojcius DM, Li S-J, Yan J. Bid-induced release of AlF/ EndoG from mitochondria causes apoptosis of macrophages during infection with Leptospira interrogans. Front Cell Infect Microbiol. 2017;7:471.

\section{Ready to submit your research? Choose BMC and benefit from:}

- fast, convenient online submission

- thorough peer review by experienced researchers in your field

- rapid publication on acceptance

- support for research data, including large and complex data types

- gold Open Access which fosters wider collaboration and increased citations - maximum visibility for your research: over $100 \mathrm{M}$ website views per year

At BMC, research is always in progress.

Learn more biomedcentral.com/submissions 